\title{
How to estimate effective dose for CT patients
}

\section{Choonsik Lee $^{1}$}

Received: 15 November 2019 / Accepted: 12 December 2019 / Published online: 4 February 2020

(C) This is a U.S. government work and not under copyright protection in the U.S.; foreign copyright protection may apply 2020

\section{Key Points}

- Rehani et al provide important insight into the status quo of CT dose and call an urgent attention to the high-dose group receiving over $100 \mathrm{mSv}$.

- It is crucial to clearly understand the calculation algorithm of effective dose behind the CT dose reporting systems and potential uncertainties.

Computed tomography $(\mathrm{CT})$ provides patients with unarguable benefit in modern medicine, which mostly outweigh the risks of adverse health effects. However, concerns about late effects from the radiation dose modality still remain particularly for younger patients. Several large-scale epidemiological studies have reported direct evidence of subsequent cancer risk after CT scans $[1,2]$. In responding to those concerns, a great deal of efforts have been made to reduce radiation dose in CT scans and to educate clinical staff involved in scanning procedures about its potential risks. It is now important to evaluate the expected changes of the dose trend and to identify the possible high-dose group for large-scale patient populations.

The paper by Rehani et al [3] provides the readers with valuable information about the cumulative radiation doses patients get from CT in the USA as well as outside the USA. The authors collected cumulative effective dose (CED) from different dose reporting systems covering 324 hospitals. They report that a total of 33,407 out of 2.5 million patients (1.33\%) received CED greater than $100 \mathrm{mSv}$ over a period of less than 5 years from recurrent $\mathrm{CT}$ scans. The numbers are much greater than anticipated from several reports in the past and in view of decreasing trend of CT dose in the past years. The study sends out a clarion call to the manufacturers of CT

This comment refers to the article available at https://doi.org/10.1007/ s00330-019-06523-y.

Choonsik Lee

choonsik.lee@nih.gov

1 Division of Cancer Epidemiology and Genetics, National Cancer Institute, National Institutes of Health, 9609 Medical Center Drive, Rockville, MD 20850, USA equipment and radiology community to take actions to address the issue of high cumulative doses to such a large number of patients. Based on their conservative estimates, they mention that nearly 0.9 million patients likely get such high dose every year globally. To make informed decisions, it is extremely important to clearly define and reliably estimate dose descriptors reported for CT scans. Many studies, including Rehani et al [3], report effective dose as a CT dose descriptor.

Effective dose was originally developed by the International Commission on Radiological Protection (ICRP) as a risk-adjusted dosimetric quantity for the management of protection against stochastic effects, principally cancer, enabling comparison of planned or received doses with dose limits, dose constraints, and reference levels expressed in the same quantity. The radiology community has gravitated towards this radiation protection quantity to describe medical radiation dose for multiple reasons: it is a single metric representing a measure of cancer risk for major radiosensitive organs throughout the patient anatomy and it is conveniently retrievable from dose monitoring systems.

Effective dose is defined by the ICRP [4] as "tissue-weighted sum of the equivalent doses in all specified tissues and organs of the body." Along with the definition, ICRP also provides the list of tissue weighting factors "representing the relative contribution of the given tissue or organ to the total health detriment resulting from uniform irradiation of the body." The tissue weighting factors are age- and genderaveraged quantities and thus are not designed for any specific individual or sub-populations such as children or women. To derive effective dose as defined in ICRP Publications, one needs absorbed dose to about 30 different organs and tissues to which tissue weighting factors are assigned. 
The most convenient way to estimate effective dose for CT patients is to use dose-length product (DLP) to effective dose conversion factors, called $k$-factors [5], which may be adopted by most $\mathrm{CT}$ dose reporting systems. Effective dose can be conveniently estimated by multiplying the conversion factors by the DLP values readily available from CT scanners. These conversion factors are pre-calculated by using computational human phantoms [6] combined with Monte Carlo radiation transport simulation of CT x-rays. Several studies have reported different sets of DLP-to-effective dose conversion factors. It is critical to consider various factors involved in the derivation of the conversion factors to make appropriate comparison among different $\mathrm{CT}$ dose studies.

First, ICRP has revised their initial tissue weighting factors reported in 1977 two times in ICRP Publication 60 [7] and 103 [4]. $k$-factors vary depending on which tissue weighting factor sets were used for their derivation, which change the resulting effective dose by up to $40 \%$ between ICRP 60 and 103 [8]. Second, $k$-factors vary depending on which computational human phantoms are used in their calculations: oldfashioned stylistic phantoms made of mathematical equations vs. realistic voxel phantoms based on radiological images of patients. $k$-factors are technically applicable to patients who have the same anatomy as the phantoms that the $k$-factors are calculated from. The $k$-factors based on voxel phantoms should be more applicable to CT patients compared with the conversion factors calculated from stylized phantoms, especially for the organs with high tissue weighting factors such as the breast, bone marrow, colon, lungs, and stomach. Effective dose estimated from stylized phantoms is reported to be different from realistic voxel phantoms by up to $40 \%$ (for head/ neck and abdomen-pelvis scans) [9]. Lastly, $k$-factors from phantoms with reference size may under- or overestimate actual effective dose delivered to underweight and overweight patients, respectively [10]. Unfortunately, currently little is known about $k$-factors and computational phantoms used in the dose monitoring systems of different vendors including those used in the study by Rehani et al. As mentioned by Rehani et al [3], there is a need in the future to have vetting of accuracy of dose estimates provided by these dose monitoring systems.

In summary, after years of effort to reduce CT dose, it is important to directly observe its impact on CT dose actually delivered to patients in a cumulative manner, something that has been largely missed in the past. As many studies use effective dose as a CT dose descriptor, it is also urgent to clearly understand the calculation algorithm of effective dose behind the CT dose reporting systems and potential uncertainties caused by several factors involved in the DLP-to-effective dose conversion factors. Rehani et al provide important insight into the status quo of $\mathrm{CT}$ dose and call an urgent attention to the high-dose group receiving over $100 \mathrm{mSv}$. If the similar high-dose group can be identified in pediatric patients and high-quality followup mechanism in place, then these data should be valuable for additional epidemiological studies on radiation effects from CT dose.

Acknowledgments This work was funded by the intramural research program of the National Institutes of Health (NIH), National Cancer Institute, Division of Cancer Epidemiology and Genetics. The contents are solely the responsibility of the authors and do not necessarily represent the official views of the NIH.

Funding information The authors state that this work has not received any funding.

\section{Compliance with ethical standards}

Guarantor The scientific guarantor of this publication is Choonsik Lee.

Conflict of interest The authors of this manuscript declare no relationships with any companies, whose products or services may be related to the subject matter of the article.

Statistics and biometry No complex statistical methods were necessary for this paper.

Informed consent Written informed consent was not required for this study because the study does not involve any human subjects or Protected Health Information (PHI).

Ethical approval Institutional Review Board approval was not required because the study does not include any human subjects or Protected Health Information (PHI).

\section{Methodology}

- Not applicable.

\section{References}

1. Pearce MS, Salotti JA, Little MP et al (2012) Radiation exposure from CT scans in childhood and subsequent risk of leukaemia and brain tumours: a retrospective cohort study. Lancet 380:499-505. https://doi.org/10.1016/S0140-6736(12)60815-0

2. Meulepas JM, Ronckers CM, Smets AMJB et al (2019) Response to Wollschläger, Blettner, and Pokora. J Natl Cancer Inst 111:10021003. https://doi.org/10.1093/jnci/djz062

3. Rehani M, Yang K, Melick E et al (2019) Patients undergoing recurrent CT scans: assessing the magnitude. Eur Radiol. https:// doi.org/10.1007/s00330-019-06523-y

4. (2007) The 2007 Recommendations of the International Commission on Radiological Protection. ICRP publication 103. Ann ICRP 37:1-332

5. McCollough C, Cody D, Edyvean S et al (2008) The measurement, reporting, and management of radiation dose in CT. Report of AAPM Task Group 23. American Association of Physicists in Medicine 1-34

6. Xu XG (2014) An exponential growth of computational phantom research in radiation protection, imaging, and radiotherapy: a review of the fifty-year history. Phys Med Biol 59:R233-R302. https://doi.org/10.1088/0031-9155/59/18/R233 
7. ICRP (1991) ICRP Publication 60: 1990 Recommendations of the International Commission on Radiological Protection. Ann ICRP $21: 1-3$

8. Christner JA, Kofler JM, McCollough CH (2010) Estimating effective dose for CT using dose-length product compared with using organ doses: consequences of adopting international commission on radiological protection publication 103 or dual-energy scanning. AJR Am J Roentgenol 194:881-889. https://doi.org/10.2214/AJR.09.3462

9. Gao Y, Quinn B, Mahmood U et al (2017) A comparison of pediatric and adult $\mathrm{CT}$ organ dose estimation methods. BMC Med Imaging 17. https://doi.org/10.1186/s12880-017-0199-3
10. Lee C, Flynn MJ, Judy PF, Cody DD, Bolch WE, Kruger RL (2017) Body size-specific organ and effective doses of chest CT screening examinations of the National Lung Screening Trial. Am J Roentgenol 208:1082-1088. https://doi.org/10.2214/AJR.16. 16979

Publisher's note Springer Nature remains neutral with regard to jurisdictional claims in published maps and institutional affiliations. 